\title{
Reduction of quantum systems with symmetry, continuous and discrete
}

$\operatorname{AUTHOR}(S)$ :

Iwai, T; Hirose, T

CITATION:

Iwai, T ... [et al]. Reduction of quantum systems with symmetry,

continuous and discrete. JOURNAL OF MATHEMATICAL PHYSICS 2002, 43(6): 2927-2947

\section{ISSUE DATE:}

2002-06

URL:

http://hdl.handle.net/2433/50280

\section{RIGHT:}

Copyright 2002 American Institute of Physics. This article may be downloaded for personal use only. Any other use requires prior permission of the author and the American Institute of Physics. 


\title{
Reduction of quantum systems with symmetry, continuous and discrete
}

\author{
Toshihiro Iwai ${ }^{\mathrm{a})}$ and Toru Hirose \\ Department of Applied Mathematics and Physics, Kyoto University, \\ Kyoto-606-8501, Japan
}

(Received 25 November 2001; accepted for publication 26 February 2002)

Reduction of dynamical systems is closely related with symmetry. The purpose of this article is to show that Fourier analysis both on compact Lie groups and on finite groups serves as a reduction procedure for quantum systems with symmetry on an equal footing. The reduction procedure is applied to systems of many identical particles lying in $\mathbf{R}^{3}$ which admit the action of a rotation group $\mathrm{SO}(3)$ and of a symmetric or permutation group. (C) 2002 American Institute of Physics.

[DOI: 10.1063/1.1473873]

\section{INTRODUCTION}

As is widely recognized, reduction of dynamical systems is closely related with symmetry. A well-known example of reduction in ordinary quantum mechanics in $\mathbf{R}^{3}$ comes from rotational symmetry. ${ }^{1}$ It gives rise to the conservation of the angular momentum, and thereby the quantum state of the system can be restricted to that with a fixed angular momentum eigenvalue. The restricted state is described by one of the spherical harmonics multiplied by a function of the radial variable. The original Schrödinger equation then reduces to provide a Schrödinger equation for the radial function. In this manner, the original quantum system reduces to a quantum system of lower degree(s) of freedom. This reduction procedure proves to be based upon Fourier analysis on the rotation group $\mathrm{SO}(3)$. The reason why Fourier analysis on $\mathrm{SO}(3)$ is referred to, instead of that on $S^{2}$ with spherical harmonics, is that Fourier analysis on $\mathrm{SO}(3)$ reduces to that on $S^{2}$ through the bundle structure $\mathrm{SO}(3) \rightarrow S^{2}$, when $\mathrm{SO}(3)$ acts on $\mathbf{R}^{3}$.

As for discrete symmetry, systems of many identical particles admit symmetry by the action of symmetric (or permutation) groups, that is, symmetry of particle exchanges. A point to make here is that the particles are not assumed to be placed at vertices of regular polyhedrons, but free to spread in the space. The center-of-mass system for $N$ identical particles is actually shown to admit the action of the symmetric group $S_{N}$. Fourier analysis on finite groups will work well in reducing the quantum system of identical particles. However, the reduction by a finite group does not mean that of degrees of freedom, but a reduction to "eigenstates" for the symmetric group.

A key idea to reduction procedure is the Peter-Weyl theorem ${ }^{2,3}$ on unitary irreducible representations of compact Lie groups and of finite groups, both of which are stated in the same manner. The Peter-Weyl theorem says that matrix elements of all the inequivalent irreducible unitary representations provide a basis of Fourier analysis on the group in question. The purpose of this article is to show that Fourier analysis both on compact Lie groups and on finite groups serves as a reduction procedure for quantum systems with symmetry, continuous and discrete, on an equal footing.

The fact that the Peter-Weyl theorem on compact Lie groups serves as a reduction procedure for quantum systems has been already stated and applied, in a previous paper, ${ }^{4}$ to many-particle systems. To understand how the Peter-Weyl theorem comes to be associated with reduction procedure for many-particle systems, one has to review geometric method for many-particle systems. For a long period before a bundle picture was introduced in the study of many-particle

${ }^{a}$ Electronic mail: iwai@amp.i.kyoto-u.ac.jp 
systems, a vain effort had been made to separate rotational and vibrational motions. However, the separation of them was shown to be impossible by A. Guichardet ${ }^{5}$ by the use of the connection theory or gauge theory applied to the center-of-mass system which is viewed as a principal fiber bundle with structure group $\mathrm{SO}(3)$, if collinear configurations of particles are gotten rid of. With this constraint taken into account, reduction procedure was described in the bundle picture. ${ }^{6}$ Since then, classical and quantum mechanics for many-particle systems have been studied in the bundle picture. $^{7-14}$

A question has been kept unsettled as to how the collinear configurations should be treated in the study of reduction procedure. An answer to this question is brought about when the problem is put in a generic setting. ${ }^{4}$ Since the center-of-mass system admits an $\mathrm{SO}(3)$ action, a geometric setting to start with is simply that a manifold $M$ is given on which a compact Lie group $G$ acts. The action of $G$ is not assumed to be free, so that $M$ is not made into a fiber bundle in general. Though the bundle picture fails to work, the theory of unitary representations of compact Lie groups works well on the space, $L^{2}(M)$, of square integrable functions on $M$. By an effective use of the Peter-Weyl theorem, $L^{2}(M)$ is decomposed into a series of subspaces, each of which is isomorphic with the space of equivariant functions taking values in a representation space, and may be viewed as the space of eigenstates assigned by the parameter, like an angular momentum eigenvalue, characterizing the representation chosen. If a given Hamiltonian is $G$-invariant, the original quantum system reduces to a system on the space of equivariant functions, which may be called a reduced system actually. The question mentioned above is now solved. In fact, the reduction procedure in this sense can be applied to many-particle systems without excluding collinear configurations. In this stage of reduction, we have not taken up a bundle picture, yet. If the action of $G$ is free furthermore, the reduction procedure can be described in the bundle picture. In fact, $M$ is then made into a principal bundle, $M \rightarrow M / G$, and the reduced system is brought into one-to-one correspondence with a quantum system defined on a complex vector bundle associated with the principal bundle $M \rightarrow M / G{ }^{10}$

A review article by Littlejohn and Reinsch ${ }^{15}$ is of great help in studying quantum mechanics of many-particle systems in the bundle picture. A lecture note by Ezra ${ }^{16}$ is a unifying survey of rotation, reflection and identical particle symmetry in molecules before the introduction of the bundle theory in many-particle systems.

This article is organized as follows: Section II contains a brief review of the reduction of quantum systems by a compact Lie group on the basis of the study in Ref. 4. Section III is devoted to the study of the reduction by a finite group. The reduction procedure will run in parallel with that by a compact Lie group. Section IV contains examples. To a better comprehension, the reduction procedure is performed for quantum systems on $L^{2}\left(\mathbf{R}^{3}\right)$ with $\mathrm{SO}(3)$ symmetry. As is stated in the beginning of this section, Fourier analysis on $\mathrm{SO}(3)$ reduces to that on $S^{2}$ according to the bundle structure $\mathrm{SO}(3) \rightarrow S^{2}$, and thereby the quantum system on $L^{2}\left(\mathbf{R}^{3}\right)$ will reduce to a series of systems defined on the closed half line $\{r \in \mathbf{R} \mid r \geqslant 0\}$ with $r$ the radial variable. Boundary conditions for wave functions at $r=0$ are also analyzed by the use of the group theory. Section $\mathrm{V}$ centers on the application of the reduction procedure to systems of $N$ identical particles. The reduction procedures with both a compact Lie group $\mathrm{SO}(3)$ and a symmetric group $S_{N}$, a discrete finite group, are performed simultaneously. Matrix representations of $S_{3}$ and $S_{4}$ will be given explicitly, which act on the center-of-mass systems for three and four particles, respectively.

\section{REDUCTION BY A COMPACT LIE GROUP}

We put the problem of reduction of quantum systems with symmetry in a general setting. This section is a review from Ref. 4. Let $M$ be a manifold on which a compact Lie group $G$ acts. Let $\mu_{M}$ be a $G$-invariant measure on $M$. The space $L^{2}(M)$ of square integrable functions on $M$ is the Hilbert space that we take as the space of wave functions. The inner product of functions on $M$ is denoted, as usual, by 


$$
\left\langle f_{1}, f_{2}\right\rangle_{L^{2}(M)}=\int_{M} \overline{f_{1}(x)} f_{2}(x) d \mu_{M}(x)
$$

The group $G$ is represented unitarily in $L^{2}(M)$ through

$$
(U(g) f)(x)=f\left(g^{-1} x\right), \quad g \in G, x \in M .
$$

By the use of the representation $g \mapsto U(g)$, one can decompose $L^{2}(M)$ into a direct sum of subspaces. Before describing the decomposition, we have to make a brief review of the PeterWeyl theorem on unitary representations of compact Lie groups.

Let $\mu_{G}$ and $L^{2}(G)$ denote the normalized invariant measure on $G$ and the space of square integrable functions on $G$ with respect to $\mu_{G}$, respectively. Let $\left(\mathcal{H}^{\chi}, \rho^{\chi}\right)$ be unitary irreducible representations of $G$, where $\chi$ ranges over all the inequivalent unitary irreducible representations. We denote by $\rho_{i j}^{\chi}$ the matrix elements of the representation $\rho^{\chi}$ with respect to some orthonormal basis of the representation space $\mathcal{H}^{\chi}$, where $i, j=1, \ldots, d_{\chi}$, and $d_{\chi}=\operatorname{dim} \mathcal{H}^{\chi}$. The Peter-Weyl theorem $^{2}$ states that the set of all the matrix elements $\left\{\sqrt{d_{\chi}} \rho_{i j}^{\chi}\right\}_{\chi, i, j}$ form a complete orthonormal system in $L^{2}(G)$. Then any function $\varphi$ in $L^{2}(G)$ is expanded into a Fourier series:

$$
\varphi(h)=\sum_{\chi} d_{\chi} \sum_{i, j} \rho_{i j}^{\chi}(h) \int_{G} \overline{\rho_{i j}^{\chi}(g)} \varphi(g) d \mu_{G}(g)=\sum_{\chi} d_{\chi} \sum_{i} \int_{G} \rho_{i i}^{\chi}(g) \varphi\left(g^{-1} h\right) d \mu_{G}(g) .
$$

This theorem can be used to find a Fourier series expansion of a function on $M$. Given a function $f \in L^{2}(M)$, we may view $f(h x)$ as a function of $h \in G$, if $x \in M$ is fixed arbitrarily. We may write this function as $f_{x}$, so that $f_{x}(h):=f(h x)$ for $h \in G$. For $\varphi=f_{x}$, Eq. (3) provides

$$
f(h x)=\sum_{\chi} d_{\chi} \sum_{i} \int_{G} \rho_{i i}^{\chi}(g) f\left(g^{-1} h x\right) d \mu_{G}(g) .
$$

In particular, for $h=e$, this formula gives a Fourier series expansion of $f$ :

$$
f(x)=\sum_{\chi} d_{\chi} \sum_{i} \int_{G} \rho_{i i}^{\chi}(g) f\left(g^{-1} x\right) d \mu_{G}(g) .
$$

This expansion suggests we define operators $P_{i}^{\chi}$ on $L^{2}(M)$ to be

$$
P_{i}^{\chi}:=d_{\chi} \int_{G} \rho_{i i}^{\chi}(g) U(g) d \mu_{G}(g)
$$

Then, a straightforward calculation shows that

$$
\left(P_{i}^{\chi}\right)^{\dagger}=P_{i}^{\chi}, \quad P_{i}^{\chi} P_{j}^{\chi^{\prime}}=\delta^{\chi \chi^{\prime}} \delta_{i j} P_{i}^{\chi}
$$

Further, the Fourier series expansion (5) means that

$$
\sum_{\chi} \sum_{i} P_{i}^{\chi}=\operatorname{id}_{L^{2}(M)}
$$

where $\operatorname{id}_{L^{2}(M)}$ denotes the identity map of $L^{2}(M)$. Equations (7) and (8) implies that the set $\left\{P_{i}^{X}\right\}_{\chi, i}$ forms a family of orthogonal projection operators and provides a resolution of unity. Hence one has the orthogonal decomposition of $L^{2}(M)$,

$$
L^{2}(M)=\bigoplus_{\chi} \bigoplus_{i=1}^{d_{\chi}} \operatorname{Im} P_{i}^{\chi}
$$


Moreover, we define the operators

$$
P_{i j}^{\chi}:=d_{\chi} \int_{G} \rho_{i j}^{\chi}(g) U(g) d \mu_{G}(g),
$$

which prove to satisfy that

$$
\left(P_{i j}^{\chi}\right)^{\dagger}=P_{j i}^{\chi}, \quad P_{i j}^{\chi} P_{k \ell}^{\chi^{\prime}}=\delta^{\chi \chi^{\prime}} \delta_{j k} P_{i \ell}^{\chi} .
$$

In particular, from $P_{i i}^{\chi}=P_{i}^{\chi}$ along with (11), one has

$$
P_{i j}^{\chi} P_{j}^{\chi}=P_{i}^{\chi} P_{i j}^{\chi}, \quad\left(P_{i j}^{\chi}\right)^{\dagger} P_{i}^{\chi}=P_{j}^{\chi}\left(P_{i j}^{\chi}\right)^{\dagger},
$$

and further

$$
\left(P_{i j}^{\chi}\right)^{\dagger} P_{i j}^{\chi}=P_{j}^{\chi}, \quad P_{i j}^{\chi}\left(P_{i j}^{\chi}\right)^{\dagger}=P_{i}^{\chi} .
$$

From (12) and (13), it follows that when restricted to $\operatorname{Im} P_{j}^{\chi}$ the operator $P_{i j}^{\chi}$ provides a unitary isomorphism

$$
P_{i j}^{\chi}: \operatorname{Im} P_{j}^{\chi} \rightarrow \operatorname{Im} P_{i}^{\chi}, \quad i, j=1, \ldots, d_{\chi} .
$$

Furthermore, we can show that the operators $P_{i j}^{X}$ and $U(g)$ are composed to give

$$
P_{i j}^{\chi} U(g)=\sum_{k} \overline{\rho_{j k}^{\chi}(g)} P_{i k}^{\chi}, \quad U(g) P_{i j}^{\chi}=\sum_{k} \overline{\rho_{k i}^{\chi}(g)} P_{k j}^{\chi}
$$

We now denote by $\mathcal{H}^{\chi} \otimes L^{2}(M)$ the space of $\mathcal{H}^{\chi}$-valued square integrable functions on $M$. The inner product in $\mathcal{H}^{\chi} \otimes L^{2}(M)$ is defined by

$$
\langle\psi, \phi\rangle_{\mathcal{H} X \otimes L^{2}(M)}=\int_{M}(\psi(x), \phi(x)) d \mu_{M}(x), \quad \psi, \phi \in \mathcal{H}^{\chi} \otimes L^{2}(M),
$$

where $(\psi(x), \phi(x))$ denotes the inner product of $\psi(x)$ and $\phi(x)$ in $\mathcal{H}^{\chi}$. The second equation of (15) then implies that the map $E_{j}^{\chi}: L^{2}(M) \rightarrow \mathcal{H}^{\chi} \otimes L^{2}(M)$ defined by

$$
E_{j}^{\chi}=\frac{1}{\sqrt{d_{\chi}}}\left(P_{1 j}^{\chi}, P_{2 j}^{\chi}, \ldots, P_{d_{\chi} j}^{\chi}\right)^{T}
$$

the superscript $T$ denoting the transpose, satisfies

$$
U\left(g^{-1}\right) E_{j}^{\chi}=\rho^{\chi}(g) E_{j}^{\chi}, \quad g \in G,
$$

which implies that $\mathcal{H}^{\chi}$-valued functions $E_{j}^{\chi} f$ with $f \in L^{2}(M)$ are subject to the transformation

$$
\left(E_{j}^{\chi} f\right)(g x)=\rho^{\chi}(g)\left(E_{j}^{\chi} f\right)(x), \quad g \in G .
$$

Put another way, the $\mathcal{H}^{\chi}$-valued functions $E_{j}^{\chi} f$ are $\rho^{\chi}$-equivariant functions. We here define the space of $\mathcal{H}^{\chi}$-valued $\rho^{\chi}$-equivariant square integrable functions to be

$$
L^{2}\left(M ; \mathcal{H}^{\chi}\right)^{G}=\left\{\psi \in \mathcal{H}^{\chi} \otimes L^{2}(M) \mid \psi(g x)=\rho^{\chi}(g) \psi(x), g \in G, x \in M\right\} .
$$

We then observe from (19) that the operator $E_{j}^{\chi}$ is a map $L^{2}(M) \rightarrow L^{2}\left(M ; \mathcal{H}^{\chi}\right)^{G}$. The adjoint operator $\left(E_{j}^{\chi}\right)^{\dagger}: L^{2}\left(M ; \mathcal{H}^{\chi}\right)^{G} \rightarrow L^{2}(M)$ is defined, of course, through 


$$
\left\langle\psi, E_{j}^{\chi} f\right\rangle_{\mathcal{H} \chi \otimes L^{2}(M)}=\left\langle\left(E_{j}^{\chi}\right)^{\dagger} \psi, f\right\rangle_{L^{2}(M)}, \quad \psi \in L^{2}\left(M ; \mathcal{H}^{\chi}\right)^{G}, f \in L^{2}(M) .
$$

We note here that components of $\rho^{\chi}$-equivariant functions satisfy

$$
P_{i j}^{\chi} \psi_{j}=\psi_{i} \quad \text { for } \quad \psi=\left(\psi_{i}\right) \in L^{2}\left(M ; \mathcal{H}^{\chi}\right)^{G} .
$$

Then, from the definition of $\left(E_{j}^{\chi}\right)^{\dagger}$, it follows that

$$
\left(E_{j}^{\chi}\right)^{\dagger} \psi=\sqrt{d_{\chi}} \psi_{j} \quad \text { for } \quad \psi=\left(\psi_{i}\right) \in L^{2}\left(M ; \mathcal{H}^{\chi}\right)^{G} .
$$

Further, from (22) and (23), one can easily show that

$$
\left(E_{j}^{\chi}\right)^{\dagger} E_{j}^{\chi}=P_{j}^{\chi}, \quad E_{j}^{\chi}\left(E_{j}^{\chi}\right)^{\dagger}=\mathrm{id}_{L^{2}(M ; \mathcal{H} X)^{G}},
$$

which implies that when restricted to $\operatorname{Im} P_{j}^{\chi}$ the map $E_{j}^{\chi}$ provides a unitary isomorphism

$$
E_{j}^{\chi}: \quad \operatorname{Im} P_{j}^{\chi} \rightarrow L^{2}\left(M ; \mathcal{H}^{\chi}\right)^{G}, \quad j=1, \ldots, d_{\chi},
$$

so that all $\operatorname{Im} P_{j}^{\chi}, j=1, \ldots, d_{\chi}$, are unitarily isomorphic to one another.

Forming the direct sum of $d_{\chi}$ copies of $L^{2}\left(M ; \mathcal{H}^{\chi}\right)^{G}$, we obtain

$$
\bigoplus_{j=1}^{d_{\chi}} \operatorname{Im} P_{j}^{\chi} \cong\left(\mathcal{H}^{\chi}\right)^{*} \otimes L^{2}\left(M ; \mathcal{H}^{\chi}\right)^{G}
$$

From (9) and (26), $L^{2}(M)$ is decomposed, in conclusion, into

$$
L^{2}(M) \cong \bigoplus_{\chi}\left(\left(\mathcal{H}^{\chi}\right)^{*} \otimes L^{2}\left(M ; \mathcal{H}^{\chi}\right)^{G}\right) .
$$

We are now in a position to describe a method for reducing quantum systems with symmetry. Let $\hat{H}$ be a Hamiltonian operator acting on a dense domain in $L^{2}(M)$. We assume that $\hat{H}$ and $U(g)$ commute for any $g \in G$. Then $\hat{H}$ and $P_{i}^{\chi}$ also commute, so that the subspace $\operatorname{Im} P_{i}^{\chi}$ is invariant under $\hat{H}$. This implies that the quantum system $\left(L^{2}(M), \hat{H}\right)$ reduces to a series of subsystems $\left(\operatorname{Im} P_{i}^{\chi}, \hat{H}\right)$ or equivalently to $\left(L^{2}\left(M ; \mathcal{H}^{\chi}\right)^{G}, \operatorname{id}_{\mathcal{H} \chi} \otimes \hat{H}\right)$, where $\operatorname{id}_{\mathcal{H}_{\chi} \otimes \hat{H}}$ means that $\mathcal{H}^{X}$-valued functions are operated componentwise with $\hat{H}$. The assumption we have used so far is that $M$ carries the $G$-invariant measure $\mu_{M}$. To give an example of $\hat{H}$ explicitly, we now assume that $M$ is endowed with a Riemannian metric and that $G$ acts on $M$ by isometry. We take $d \mu_{M}$ as the volume element formed from the metric. As usual, we take $\hat{H}=-\frac{1}{2} \Delta_{M}+v$, where $\Delta_{M}$ is the Laplacian on $M$ and $v$ is a $G$-invariant function on $M$. Since this Hamiltonian is $G$-invariant, the quantum system $\left(L^{2}(M), \hat{H}\right)$ reduces to $\left(L^{2}\left(M ; \mathcal{H}^{\chi}\right)^{G}, \operatorname{id}_{\mathcal{H}_{\chi}} \otimes \hat{H}\right)$.

If the action of the compact Lie group $G$ is free furthermore, $M$ is made into a fiber bundle $M \rightarrow M / G$ with structure group $G$. Then, as is well known, the space of the $\mathcal{H}^{X}$-valued $\rho^{X}$-equivariant functions is in one-to-one correspondence with the space of sections in the complex vector bundle associated with the principal bundle $M \rightarrow M / G$. According to this, the Hamiltonian operator $\operatorname{id}_{\mathcal{H}} \otimes \hat{H}$ gives rise to a Hamiltonian operator $\hat{H}^{\chi}$ acting on the space of sections. Let $\Gamma_{\chi}^{2}(M / G)$ denote the space of square integrable sections in the complex vector bundle. The reduced quantum system $\left(L^{2}\left(M ; \mathcal{H}^{\chi}\right)^{G}\right.$, id $\left._{\mathcal{H}} \otimes \hat{H}\right)$ now determines a quantum system $\left(\Gamma_{\chi}^{2}(M / G), \hat{H}^{\chi}\right)$. To find $\hat{H}^{\chi}$ in an explicit manner, we need a further study, which we do not touch upon here (see Ref. 4 for details).

If the action of $G$ is not free, the orbit space $M / G$ is not a manifold, and hence the bundle picture of reduction procedure stated above fails to work. However, the reduction to 
$\left(L^{2}\left(M ; \mathcal{H}^{\chi}\right)^{G}, \mathrm{id}_{\mathcal{H}} \otimes \hat{H}\right)$ remains to be the case. In some cases, orbit spaces become manifolds with boundary. For example, for $M=\mathbf{R}^{3}$ and $G=\mathrm{SO}(3)$, the orbit space $M / G$ is a closed half line $\{r \in \mathbf{R} \mid r \geqslant 0\}$. This will be treated in Sec. IV.

\section{REDUCTION BY A FINITE GROUP}

We wish to show that the reduction procedure will work as well if we take finite groups in place of compact Lie groups. We start with a review of the Peter-Weyl theorem for finite groups. Let $H$ be a finite group. Let $\pi_{i j}^{\chi}$ denote the matrix elements of the representation $\left(\mathcal{K}^{\chi}, \pi^{\chi}\right)$ of $H$, where $i, j=1, \ldots, d^{\chi}$ with $d^{\chi}=\operatorname{dim} \mathcal{K}^{\chi}$, and $\chi$ ranges all the inequivalent unitary irreducible representations. The Peter-Weyl theorem for finite groups ${ }^{3}$ says that all the matrix elements $\left\{\pi_{i j}^{\chi}\right\}_{\chi, i, j}$ form a complete orthogonal set in $L^{2}(H)$. The inner product for $\varphi, \psi \in L^{2}(H)$ is defined, as usual, to be

$$
\langle\varphi, \psi\rangle_{L^{2}(H)}=\sum_{g \in H} \overline{\varphi(g)} \psi(g) .
$$

The orthogonality of the matrix elements is expressed as

$$
\frac{d^{\chi}}{|H|} \sum_{g \in H} \overline{\pi_{i j}^{\chi}(g)} \pi_{i^{\prime} j^{\prime}}^{\chi^{\prime}}(g)=\delta_{\chi \chi^{\prime}} \delta_{i i^{\prime}} \delta_{j j^{\prime}},
$$

where $|H|=\# H$, the order of $H$. The Fourier inversion formula then holds to provide

$$
\varphi(g)=\frac{1}{|H|} \sum_{\chi} d^{\chi} \sum_{1 \leqslant i, j \leqslant d^{\chi}} \pi_{i j}^{\chi}(g)\left\langle\pi_{i j}^{\chi}, \varphi\right\rangle_{L^{2}(H)}=\frac{1}{|H|} \sum_{\chi} d^{\chi} \sum_{j=1}^{d^{\chi}} \sum_{k \in H} \overline{\pi_{j j}^{\chi}(k)} \varphi(g k) .
$$

Let $M$ be a manifold which admits a right action of $H$, where the right action means that $x(g h)=(x g) h$ for $x \in M$ and $g, h \in H$. Let $L^{2}(M)$ denote the space of square integrable functions on $M$, where the measure $\mu_{M}$ on $M$ is assumed to be invariant under $H$. The $H$ is unitarily represented in $L^{2}(M)$ through

$$
(V(g) f)(x)=f(x g), \quad x \in M, \quad g \in H .
$$

Applying the Peter-Weyl formula for $f(x g)$ with $x \in M$ arbitrarily fixed, one obtains

$$
f(x g)=\frac{1}{|H|} \sum_{\chi} d^{\chi} \sum_{1 \leqslant i, j \leqslant d^{X}} \pi_{i j}^{\chi}(g) \sum_{h \in H} \overline{\pi_{i j}^{\chi}(h)} f(x h)=\sum_{\chi} \sum_{j=1}^{d^{\chi}} \frac{d^{\chi}}{|H|} \sum_{k \in H} \overline{\pi_{j j}^{\chi}(k)} f(x g k) .
$$

In particular, for $g=e$, this formula gives a Fourier series expansion of $f$,

$$
f(x)=\sum_{\chi} \sum_{j=1}^{d^{\chi}} \frac{d^{\chi}}{|H|} \sum_{k \in H} \overline{\pi_{j j}^{\chi}(k)} f(x k) .
$$

This suggests we define operators $Q_{j}^{\chi}$ on $L^{2}(M)$ by

$$
Q_{j}^{\chi}=\frac{d^{\chi}}{|H|} \sum_{k \in H} \overline{\pi_{j j}^{\chi}(k)} V(k) .
$$

A straightforward calculation shows that

$$
\left(Q_{i}^{\chi}\right)^{\dagger}=Q_{i}^{\chi}, \quad Q_{i}^{\chi} Q_{i^{\prime}}^{\chi^{\prime}}=\delta^{\chi \chi^{\prime}} \delta_{i i^{\prime}} Q_{i}^{\chi},
$$


which means that $Q_{j}^{\chi}$,s form a family of mutually orthogonal projection operators. The Fourier series expansion (33) is now put in the form

$$
f(x)=\sum_{\chi} \sum_{j=1}^{d^{\chi}}\left(Q_{j}^{\chi} f\right)(x),
$$

which implies that $L^{2}(M)$ is decomposed into

$$
L^{2}(M)=\bigoplus_{\chi} \bigoplus_{j=1}^{d^{\chi}} \operatorname{Im} Q_{j}^{\chi} .
$$

We now define operators $Q_{i j}^{X}$ on $L^{2}(M)$ to be

$$
Q_{i j}^{\chi}=\frac{d^{\chi}}{|H|} \sum_{k \in H} \overline{\pi_{i j}^{\chi}(k)} V(k) .
$$

A straightforward calculation shows that these operators have the properties

$$
\left(Q_{i j}^{\chi}\right)^{\dagger}=Q_{j i}^{\chi}, \quad Q_{i j}^{\chi} Q_{i^{\prime} j^{\prime}}^{\chi^{\prime}}=\delta^{\chi \chi^{\prime}} \delta_{j i^{\prime}} Q_{i j^{\prime}}^{\chi} .
$$

In particular, from $Q_{i i}^{\chi}=Q_{i}^{\chi}$ together with (39), one verifies that

$$
Q_{i j}^{\chi} Q_{j}^{\chi}=Q_{i}^{\chi} Q_{i j}^{\chi}=Q_{i j}^{\chi}
$$

and further that

$$
\left(Q_{i j}^{\chi}\right)^{\dagger} Q_{i j}^{\chi}=Q_{j}^{\chi}, \quad Q_{i j}^{\chi}\left(Q_{i j}^{\chi}\right)^{\dagger}=Q_{i}^{\chi} .
$$

From (40) and (41), it turns out that when restricted to $\operatorname{Im} Q_{j}^{\chi}$ the map $Q_{i j}^{\chi}$ provides a unitary isomorphism,

$$
Q_{i j}^{\chi}: \operatorname{Im} Q_{j}^{\chi} \sim \operatorname{Im} Q_{i}^{\chi}, \quad i, j=1, \ldots, d^{\chi} .
$$

We can also verify that $Q_{i j}^{\chi}$ and $V(g)$ are composed to give

$$
Q_{i j}^{\chi} V(g)=\sum_{\ell} \pi_{j \ell}^{\chi}(g) Q_{i \ell}^{\chi}, \quad V(g) Q_{i j}^{\chi}=\sum_{\ell} \pi_{\ell i}^{\chi}(g) Q_{\ell j}^{\chi}
$$

We here denote by $\mathcal{K}^{\chi} \otimes L^{2}(M)$ the space of $\mathcal{K}^{\chi}$-valued square integrable functions on $M$. Then the second equation of (43) implies that the operators $F_{j}^{\chi}: L^{2}(M) \rightarrow \mathcal{K}^{\chi} \otimes L^{2}(M)$ defined to be

$$
F_{j}^{\chi}:=\frac{1}{\sqrt{d^{\chi}}}\left(Q_{1 j}^{\chi}, \ldots, Q_{d \chi_{j}}^{\chi}\right)^{T}
$$

have the property

$$
V(g) F_{j}^{\chi}=\pi^{\chi}(g)^{T} F_{j}^{\chi}, \quad g \in H .
$$

This implies that for $f \in L^{2}(M)$ the $\mathcal{K}^{\chi}$-valued function $F_{j}^{\chi} f$ is subject to the transformation

$$
\left(F_{j}^{\chi} f\right)(x g)=\pi^{\chi}(g)^{T}\left(F_{j}^{\chi} f\right)(x), \quad g \in H .
$$


We may say that the $\mathcal{K}^{\chi}$-valued function $F_{j}^{\chi} f$ is $\pi^{\chi}$-equivariant. We here define the space of $\mathcal{K}^{X}$-valued square integrable $\pi^{\chi}$-equivariant functions to be

$$
L^{2}(M ; \mathcal{K} \chi)^{H}=\left\{\psi \in \mathcal{K} \chi \otimes L^{2}(M) \mid \psi(x g)=\pi^{\chi}(g)^{T} \psi(x), \quad g \in H, x \in M\right\} .
$$

Then Eq. (46) shows that the operator $F_{j}^{\chi}$ is a map $L^{2}(M) \rightarrow L^{2}\left(M ; \mathcal{K}^{\chi}\right)^{H}$. The adjoint operator $\left(F_{j}^{\chi}\right)^{\dagger}: L^{2}(M ; \mathcal{K} \chi)^{H} \rightarrow L^{2}(M)$ is defined through

$$
\left\langle\psi, F_{j}^{\chi} f\right\rangle_{\mathcal{K} \chi \otimes L^{2}(M)}=\left\langle\left(F_{j}^{\chi}\right)^{\dagger} \psi, f\right\rangle_{L^{2}(M)}, \quad \psi \in L^{2}\left(M ; \mathcal{K}^{\chi}\right)^{H}, f \in L^{2}(M) .
$$

We notice here that components $\psi_{i}$ of $\psi \in L^{2}\left(M ; \mathcal{K}^{\chi}\right)^{H}$ are related by $Q_{i j}^{\chi}$ :

$$
Q_{i j}^{\chi} \psi_{j}=\psi_{i}, \quad i, j=1, \ldots, d^{\chi} .
$$

Then, writing out the defining equation of $\left(F_{j}^{\chi}\right)^{\dagger}$, one obtains

$$
\left(F_{j}^{\chi}\right)^{\dagger} \psi=\sqrt{d^{\chi}} \psi_{j} \text { for } \quad \psi=\left(\psi_{i}\right) \in L^{2}\left(M ; \mathcal{K}^{\chi}\right)^{H} .
$$

Now it is easy to verify that

$$
\left(F_{j}^{\chi}\right)^{\dagger} F_{j}^{\chi}=Q_{j}^{\chi}, \quad F_{j}^{\chi}\left(F_{j}^{\chi}\right)^{\dagger}=\mathrm{id}_{L^{2}(M ; \mathcal{K} X)^{H}} .
$$

This implies that when restricted to $\operatorname{Im} Q_{j}^{\chi}$ the operator $F_{j}^{\chi}$ provides a unitary isomorphism

$$
F_{j}^{\chi}: \operatorname{Im} Q_{j}^{\chi} \stackrel{\sim}{\rightarrow} L^{2}(M ; \mathcal{K} \chi)^{H}, \quad j=1, \ldots, d^{\chi},
$$

so that all $\operatorname{Im} Q_{j}^{\chi}, j=1, \ldots, d^{\chi}$, are unitarily isomorphic to one another.

Forming the direct sum of $d^{\chi}$ copies of $L^{2}\left(M ; \mathcal{K}^{\chi}\right)^{H}$, we obtain the isomorphism

$$
\bigoplus_{j} \operatorname{Im} Q_{j}^{\chi} \cong\left(\mathcal{K}^{\chi}\right)^{*} \otimes L^{2}\left(M ; \mathcal{K}^{\chi}\right)^{H},
$$

and further, from (37),

$$
L^{2}(M) \cong \bigoplus_{\chi}\left(\left(\mathcal{K}^{\chi}\right)^{*} \otimes L^{2}\left(M ; \mathcal{K}^{\chi}\right)^{H}\right)
$$

Reduction procedure for quantum systems with discrete symmetry is quite the same as that for those with compact Lie group symmetry. If the Hamiltonian $\hat{H}$ is invariant under the $H$ action, the original system $\left(L^{2}(M), \hat{H}\right)$ reduces to a series of subsystems $\left(\operatorname{Im} Q_{j}^{\chi}, \hat{H}\right)$ and then equivalently to $\left(L^{2}\left(M ; \mathcal{K}^{\chi}\right)^{H}, \operatorname{id}_{\mathcal{K} \chi} \otimes \hat{H}\right)$.

\section{EXAMPLES}

In this section, we give examples of the reduction procedure discussed in Secs. II and III. As the group $\mathrm{SO}(3)$ is the most frequently used compact Lie group in ordinary quantum mechanics, we first perform the reduction procedure with $G=\mathrm{SO}(3)$ and $M=\mathbf{R}^{3}$. In this case, one has matrix elements $D_{m m^{\prime}}^{\ell}$ for $\rho_{i j}^{\chi}$, where $\ell=0,1,2, \ldots,|m|,\left|m^{\prime}\right| \leqslant \ell$, and $d_{\chi}=2 \ell+1$. Then the Fourier series expansion (5) is put in the form

$$
f(\mathbf{x})=\sum_{\ell=0}^{\infty} \sum_{|m| \leqslant \ell}(2 \ell+1) \int_{\mathrm{SO}(3)} \overline{D_{m m}^{\ell}(h)} f(h \mathbf{x}) d \mu(h), \quad \mathbf{x} \in \mathbf{R}^{3},
$$

where $d \mu(h)$ is the invariant measure on $\mathrm{SO}(3)$ and expressed in terms of the Euler angles $h$ $=e^{\phi \hat{\mathbf{e}}_{3}} e^{\theta \hat{\mathbf{e}}_{2}} e^{\psi \hat{\mathbf{e}}_{3}}$ as 


$$
d \mu(h)=\frac{1}{2 \pi^{2}} \sin \theta d \theta d \phi d \psi \quad \text { with } \quad \int_{\mathrm{SO}(3)} d \mu(h)=1
$$

where $\mathbf{e}_{k}, k=1,2,3$, are the standard basis of $\mathbf{R}^{3}$ and $\hat{\mathbf{e}}_{k}$ denote the $3 \times 3$ matrices defined by $\hat{\mathbf{e}}_{k} \mathbf{a}=\mathbf{e}_{k} \times \mathbf{a}$ for $\mathbf{a} \in \mathbf{R}^{3}$. We wish to show that Eq. (55) provides actually a Fourier series expansion in terms of the spherical harmonics. To this end, we are to write out the integrals on the right-hand side of (55). Let $|\mathbf{x}|=r$ and set $\mathbf{x}=r g \mathbf{e}_{3}, g \in \mathrm{SO}(3)$. Then, introducing new variable $k=h g$ $\in \mathrm{SO}(3)$, one obtains

$$
\int_{\mathrm{SO}(3)} \overline{D_{m m}^{\ell}(h)} f(h \mathbf{x}) d \mu(h)=\sum_{|n| \leqslant \ell} \int_{\mathrm{SO}(3)} \overline{D_{m n}^{\ell}(k) D_{n m}^{\ell}\left(g^{-1}\right)} f\left(r k \mathbf{e}_{3}\right) d \mu(k)
$$

We now set $k=e^{\phi^{\prime} \hat{\mathbf{e}}_{3}} e^{\theta^{\prime} \hat{\mathbf{e}}_{2}} e^{\psi^{\prime} \hat{\mathbf{e}}_{3}}$, and use the fact that the $D$-functions ${ }^{17}$ are expressed as

$$
D_{m n}^{\ell}(k)=e^{-i m \phi^{\prime}} d_{m n}^{\ell}\left(\theta^{\prime}\right) e^{-i n \psi^{\prime}}
$$

where we do not need to give the explicit expression of $d_{m n}^{\ell}\left(\theta^{\prime}\right)$, but need only to note that the $D$-functions are factorized in accordance with the Euler variables. Then the integration with respect to $d \mu(k)$ in $(57)$ is put in the form

$$
\int_{\mathrm{SO}(3)} \overline{D_{m n}^{\ell}(k)} f\left(r k \mathbf{e}_{3}\right) d \mu(k)=\frac{1}{8 \pi^{2}} \int_{0}^{2 \pi} d \psi^{\prime} e^{i n \psi^{\prime}} \int_{S^{2}} d_{m n}^{\ell}\left(\theta^{\prime}\right) e^{i m \phi^{\prime}} f\left(r k \mathbf{e}_{3}\right) \sin \theta^{\prime} d \theta^{\prime} d \phi^{\prime}
$$

Since the right-hand side of (59) vanishes if $n \neq 0$, the Fourier series expansion (55) turns out to take the form

$$
\begin{aligned}
f(\mathbf{x}) & =\sum_{\ell=0}^{\infty} \sum_{|m| \leqslant \ell}(2 \ell+1) \int_{\mathrm{SO}(3)} \overline{D_{m 0}^{\ell}(k) D_{0 m}^{\ell}\left(g^{-1}\right)} f\left(r k \mathbf{e}_{3}\right) d \mu(k) \\
& =\sum_{\ell=0}^{\infty} \sum_{|m| \leqslant \ell} \bar{Y}_{\ell m}(\theta, \phi) \int_{S^{2}} Y_{\ell m}\left(\theta^{\prime}, \phi^{\prime}\right) f\left(r k \mathbf{e}_{3}\right) \sin \theta^{\prime} d \theta^{\prime} d \phi^{\prime}
\end{aligned}
$$

where we have also set $g=e^{\phi \hat{\mathbf{e}}_{3}} e^{\theta \hat{\mathbf{e}}_{2}} e^{\psi \hat{\mathbf{e}}_{3}}$ and used the formulas that relate $D$-functions to spherical harmonics: ${ }^{16}$

$$
\overline{D_{m 0}^{\ell}(k)}=\sqrt{\frac{4 \pi}{2 \ell+1}} Y_{\ell m}\left(\theta^{\prime}, \phi^{\prime}\right), \quad \overline{D_{0 m}^{\ell}\left(g^{-1}\right)}=D_{m 0}^{\ell}(g)=\sqrt{\frac{4 \pi}{2 \ell+1}} \bar{Y}_{\ell m}(\theta, \phi),
$$

and $Y_{\ell m}(\theta, \phi)$ are given explicitly by

$$
Y_{\ell m}(\theta, \phi)=(-1)^{m} \sqrt{\frac{(2 \ell+1)(\ell-m) !}{4 \pi(\ell-m) !}} P_{\ell}^{m}(\cos \theta) e^{i m \phi}
$$

where $P_{\ell}^{m}$ are associated Legendre functions. ${ }^{16}$ We notice here that $\bar{Y}_{\ell m}(\theta, \phi)=\bar{Y}_{\ell m}\left(g \mathbf{e}_{3}\right)$ may be considered as functions on $S^{2}$. If we introduce the notation

$$
\left\langle\bar{Y}_{\ell m}, f\right\rangle_{S^{2}}=: \int_{S^{2}} Y_{\ell m}\left(\theta^{\prime}, \phi^{\prime}\right) f\left(r k \mathbf{e}_{3}\right) \sin \theta^{\prime} d \theta^{\prime} d \phi^{\prime}
$$

which is a function of $r$, the Fourier series expansion (60) is put in the form 


$$
f(\mathbf{x})=\sum_{\ell=0}^{\infty} \sum_{|m| \leqslant \ell} \bar{Y}_{\ell m}(\theta, \phi)\left\langle\bar{Y}_{\ell m}, f\right\rangle_{S^{2}}, \quad \mathbf{x}=r e^{\phi \hat{\mathbf{e}}_{3}} e^{\theta \hat{\mathbf{e}}_{2}} \mathbf{e}_{3} .
$$

Thus we have obtained a Fourier series expansion in terms of spherical harmonics. It is of great interest to view the function $f\left(r k \mathbf{e}_{3}\right)$ in (63) as a function on $\mathbf{R}_{+} \times S^{2}$ which is reduced from a function on $\mathbf{R}_{+} \times \mathrm{SO}$ (3) through the bundle projection $\mathrm{SO}(3) \rightarrow S^{2}$ realized as $k \mapsto k \mathbf{e}_{3}$.

We proceed to the projection and "transition" operators, which are defined by (6) and (10) and denoted by $P_{m}^{\ell}$ and $P_{n m}^{\ell}$, respectively, in the case of $G=\mathrm{SO}(3)$. Applied to a function $f(\mathbf{x})$ with $|\mathbf{x}|=r$ and $\mathbf{x}=r g \mathbf{e}_{3}$, the definition (10) gives rise to the function

$$
\left(P_{n m}^{\ell} f\right)(\mathbf{x})=(2 \ell+1) \int_{\mathrm{SO}(3)} D_{n m}^{\ell}(h) f\left(h^{-1} \mathbf{x}\right) d \mu(h)=\bar{Y}_{\ell n}(\theta, \phi)\left\langle\bar{Y}_{\ell m}, f\right\rangle_{S^{2}},
$$

which can be proved in a similar manner to that for bringing (55) into (60). Setting $m=n$ in the above equation results in

$$
\left(P_{m}^{\ell} f\right)(\mathbf{x})=\bar{Y}_{\ell m}(\theta, \phi)\left\langle\bar{Y}_{\ell m}, f\right\rangle_{S^{2}},
$$

which means that $\operatorname{Im} P_{m}^{\ell}$ is the space of functions which are expressed as $\bar{Y}_{\ell m}$ times functions of $r$. In particular, operating $\bar{Y}_{\ell m}$ with $P_{n m}^{\ell}$, one obtains

$$
\left(P_{n m}^{\ell} \bar{Y}_{\ell m}\right)(\theta, \phi)=\bar{Y}_{\ell n}(\theta, \phi) .
$$

Then the unitary isomorphism $P_{n m}^{\ell}: \operatorname{Im} P_{m}^{\ell} \rightarrow \operatorname{Im} P_{n}^{\ell}$ [see (14)] implies that the spaces $\operatorname{Im} P_{n}^{\ell},|n|$ $\leqslant \ell$, are isomorphic to one another as spaces of functions of $r$. In the Dirac notation, we may describe $P_{n m}^{\ell}$ and $P_{m}^{\ell}$ as

$$
P_{n m}^{\ell}=\left|\bar{Y}_{\ell n}\right\rangle\left\langle\bar{Y}_{\ell m}\left|, \quad P_{m}^{\ell}=\right| \bar{Y}_{\ell m}\right\rangle\left\langle\bar{Y}_{\ell m}\right|,
$$

respectively. Here, integration must be performed not over $\mathbf{R}^{3}$ but over $S^{2}$, if one wishes to evaluate $P_{m}^{\ell} f$, for example.

We now proceed to the map $E_{j}^{\chi}$ defined by (17). From the definition along with (68), we see that $E_{m}^{\ell}: L^{2}\left(\mathbf{R}^{3}\right) \rightarrow \mathcal{H}^{\ell} \otimes L^{2}\left(\mathbf{R}^{3}\right)$ is given by

$$
E_{m}^{\ell} f=\frac{1}{\sqrt{2 \ell+1}}\left(\begin{array}{c}
P_{\ell m}^{\ell} f \\
P_{\ell-1 m}^{\ell} f \\
\vdots \\
P_{-\ell m}^{\ell} f
\end{array}\right)=\left(\begin{array}{c}
\bar{Y}_{\ell \ell} \\
\bar{Y}_{\ell \ell-1} \\
\vdots \\
\bar{Y}_{\ell-\ell}
\end{array}\right) \frac{\left\langle\bar{Y}_{\ell m}, f\right\rangle_{S^{2}}}{\sqrt{2 \ell+1}} .
$$

According to (19), $E_{m}^{\ell} f$ must be a $D^{\ell}$-equivariant function, that is, it must satisfy the condition

$$
\left(E_{m}^{\ell} f\right)(h \mathbf{x})=D^{\ell}(h)\left(E_{m}^{\ell} f\right)(\mathbf{x}), \quad h \in \mathrm{SO}(3) .
$$

However, this can also be shown to hold from the transformation rule for the spherical harmonics,

$$
\bar{Y}_{\ell m}\left(h g \mathbf{e}_{3}\right)=\sum_{|n| \leqslant \ell} D_{m n}^{\ell}(h) \bar{Y}_{\ell n}\left(g \mathbf{e}_{3}\right), \quad h \in \mathrm{SO}(3),
$$

and from the $\mathrm{SO}(3)$ invariance of $\left\langle\bar{Y}_{\ell m}, f\right\rangle_{S^{2}}$.

We have to note here that if $h$ is in $G_{\mathbf{x}}$, the isotropy subgroup of $\mathrm{SO}(3)$ at $\mathbf{x}=r g \mathbf{e}_{3}$, Eq. (70) reduces to

$$
\left(E_{m}^{\ell} f\right)(\mathbf{x})=D^{\ell}(h)\left(E_{m}^{\ell} f\right)(\mathbf{x}), \quad h \in G_{\mathbf{x}} .
$$


Since the left-hand side of the above equation is independent of $h$, so is the right-hand side which looks dependent on $h$. However, this is not a contradiction, but rather accounts for the fact that $P_{n m}^{\ell} f\left(=\bar{Y}_{\ell n}\left\langle\bar{Y}_{\ell m}, f\right\rangle_{S^{2}}\right)$ is expressed as $\bar{Y}_{\ell n}$ times a function of $r$. The proof runs as follows: Let $h \in G_{\mathbf{x}}$ with $\mathbf{x} \neq 0$. Then $h$ must be a rotation about the $\mathbf{x}$-axis, and is expressed as

$$
h=e^{t \hat{\mathbf{x}} / r}=g e^{t \hat{\mathbf{e}}_{3}} g^{-1}, \quad \mathbf{x}=r g \mathbf{e}_{3}, \quad t \in \mathbf{R} .
$$

Put together, Eqs. (72) and (73) give rise to

$$
\left(E_{m}^{\ell} f\right)\left(r \mathbf{e}_{3}\right)=D^{\ell}\left(e^{t \hat{\mathbf{e}}_{3}}\right)\left(E_{m}^{\ell} f\right)\left(r \mathbf{e}_{3}\right) .
$$

Since $D_{n m}^{\ell}\left(e^{t \hat{\mathbf{e}}_{3}}\right)=e^{-i n t} \delta_{n m}$, the above equation implies that $2 \ell$ components of $\left(E_{m}^{\ell} f\right)\left(r \mathbf{e}_{3}\right)$ vanish:

$$
\left(P_{n m}^{\ell} f\right)\left(r \mathbf{e}_{3}\right)=0 \quad \text { if } \quad n \neq 0 .
$$

From (15) and (75), it follows that

$$
\left(P_{n m}^{\ell} f\right)(\mathbf{x})=\sum_{|k| \leqslant \ell} D_{n k}^{\ell}(g)\left(P_{k m}^{\ell} f\right)\left(r \mathbf{e}_{3}\right)=D_{n 0}^{\ell}(g)\left(P_{0 m}^{\ell} f\right)\left(r \mathbf{e}_{3}\right)
$$

We observe from (61) and (76) that $\left(P_{n m}^{\ell} f\right)(\mathbf{x})$ takes the form of $\bar{Y}_{\ell n}\left(g \mathbf{e}_{3}\right)$ times a function of $r$, $\sqrt{4 \pi /(2 \ell+1)}\left(P_{0 m}^{\ell} f\right)\left(r \mathbf{e}_{3}\right)$.

With $P_{m}^{\ell} f=\bar{Y}_{\ell m}\left\langle\bar{Y}_{\ell m}, f\right\rangle_{S^{2}}$ instead of $f$, the right-hand side of (69) is unchanged:

$$
E_{m}^{\ell} P_{m}^{\ell} f=\left(\begin{array}{c}
\bar{Y}_{\ell \ell} \\
\bar{Y}_{\ell \ell-1} \\
\vdots \\
\bar{Y}_{\ell-\ell}
\end{array}\right) \frac{\left\langle\bar{Y}_{\ell m}, f\right\rangle_{S^{2}}}{\sqrt{2 \ell+1}}
$$

This equation must be a realization of the unitary isomorphism (25), which is denoted by $E_{m}^{\ell}$ : $\operatorname{Im} P_{m}^{\ell} \rightarrow L^{2}\left(\mathbf{R}^{3} ; \mathcal{H}^{\ell}\right)^{\mathrm{SO}(3)}$ in the present case, where $\mathcal{H}^{\ell} \cong \mathbf{C}^{2 \ell+1}$. From (66) and (77), both $\operatorname{Im} P_{m}^{\ell}$ and $L^{2}\left(\mathbf{R}^{3} ; \mathcal{H}^{\ell}\right)^{\mathrm{SO}(3)}$ may be identified with the space of functions of the form $\left\langle\bar{Y}_{\ell m}, f\right\rangle_{S^{2}}$. This space can be endowed with a suitable norm. We set $\varphi_{\ell m}(r)=\left\langle\bar{Y}_{\ell m}, f\right\rangle_{S^{2}}$ for simplicity. Then, the squared norm of $P_{m}^{\ell} f=\bar{Y}_{\ell m} \varphi_{\ell m}$ is calculated as

$$
\int_{0}^{\infty} d r \int_{S^{2}} \overline{\varphi_{\ell m}(r)} \varphi_{\ell m}(r) \bar{Y}_{\ell m} Y_{\ell m} r^{2} \sin \theta d \theta d \phi=\int_{0}^{\infty}\left|\varphi_{\ell m}(r)\right|^{2} r^{2} d r
$$

Thus $L^{2}\left(\mathbf{R}^{3} ; \mathcal{H}^{\ell}\right)^{\mathrm{SO}(3)}$ can be identified with the space of functions of $r$ which are subject to the condition

$$
\int_{0}^{\infty}|\varphi(r)|^{2} r^{2} d r<+\infty
$$

To consider boundary conditions for $\varphi(r)$ at $r=0$, we now take into account the $D^{\ell}$-equivariance condition (70) at the origin. Since the isotropy subgroup at the origin is $\mathrm{SO}(3)$ itself, the $D^{\ell}$-equivariance condition (70) at the origin is expressed as

$$
\bar{Y}_{\ell n}\left(g \mathbf{e}_{3}\right) \varphi_{\ell m}(0)=\sum_{\left|m^{\prime}\right| \leqslant \ell} D_{n m^{\prime}}^{\ell}(h) \bar{Y}_{\ell m^{\prime}}\left(g \mathbf{e}_{3}\right) \varphi_{\ell}(0) \text { for } \quad \forall h \in \mathrm{SO}(3) .
$$


This implies that the vector $\left(\bar{Y}_{\ell n} \varphi_{\ell m}(0)\right)_{|n| \leqslant \ell} \in \mathcal{H}^{\ell}$ is invariant under the action of all the matrices $D^{\ell}(h), h \in \mathrm{SO}(3)$. Since the representation $D^{\ell}$ is irreducible, $\varphi_{\ell m}(0)$ must vanish if $\operatorname{dim} \mathcal{H}^{\ell} \geqslant 2$, i.e., $\ell \geqslant 1$. If $\ell=0$, then $\operatorname{dim} \mathcal{H}^{0}=1$, so that $\varphi_{\ell m}(0)$ does not need to vanish. It should be a finite value. Thus the space of square integrable functions on the closed half line $\{r \in \mathbf{R} \mid r$ $\geqslant 0\}$, as a reduced space of quantum states, should be given by

$$
\left\{\left.\varphi\left|\int_{0}^{\infty}\right| \varphi(r)\right|^{2} r^{2} d r<+\infty, \quad \varphi(0)=0\right\} \quad \text { for } \quad \ell \geqslant 1,
$$

and

$$
\left\{\left.\varphi\left|\int_{0}^{\infty}\right| \varphi(r)\right|^{2} r^{2} d r<+\infty, \varphi(r) \text { is bounded as } r \rightarrow 0\right\} \text { for } \ell=0 \text {. }
$$

The reduction procedure for quantum systems with symmetry proceeds as follows: Let $\left(L^{2}\left(\mathbf{R}^{3}\right), \hat{H}\right)$ be a quantum system with a Hamiltonian operator $\hat{H}$. Assume that $\hat{H}$ is invariant under the action of $\mathrm{SO}(3)$. According to the procedure stated in Sec. II, one obtains a reduced quantum system $\left(\operatorname{Im} P_{m}^{\ell}, \hat{H}\right)$ or $\left(L^{2}\left(\mathbf{R}^{3} ; \mathcal{H}^{\ell}\right)^{\mathrm{SO}(3)}, \operatorname{id}_{\mathcal{H}}^{\ell} \otimes \hat{H}\right)$. The space $L^{2}\left(\mathbf{R}^{3} ; \mathcal{H}^{\ell}\right)^{\mathrm{SO}(3)}$ may be identified with the $L^{2}$-space on the half line which is defined by (81) for $\ell \geqslant 1$ and by (82) for $\ell=0$.

We show that the reduced quantum system $\left(\operatorname{Im} P_{m}^{\ell}, \hat{H}\right)$ gives rise to a quantum system to be defined on the closed half line $\{r \in \mathbf{R} \mid r \geqslant 0\}$. For simplicity, we assume that the Hamiltonian operator has the form

$$
\hat{H}=-\frac{1}{2} \Delta+v(r),
$$

where $\Delta$ and $v(r)$ are the standard Laplacian on $\mathbf{R}^{3}$ and a potential function depending on $r$ $=|\mathbf{x}|$, respectively. The $\Delta$ is expressed, in terms of the spherical polar coordinates, as

$$
\Delta=\frac{1}{r^{2}} \frac{\partial}{\partial r}\left(r^{2} \frac{\partial}{\partial r}\right)+\frac{1}{r^{2}} \Lambda
$$

where $\Lambda$ is the spherical Laplacian on $S^{2}$,

$$
\Lambda=\frac{1}{\sin \theta} \frac{\partial}{\partial \theta}\left(\sin \theta \frac{\partial}{\partial \theta}\right)+\frac{1}{\sin ^{2} \theta} \frac{\partial^{2}}{\partial \phi^{2}} .
$$

Operating $P_{m}^{\ell} f=\bar{Y}_{\ell m} \varphi_{\ell m}$ with $\hat{H}$, one obtains

$$
\hat{H} P_{m}^{\ell} f=\bar{Y}_{\ell m}\left(-\frac{1}{2} \frac{1}{r^{2}} \frac{\partial}{\partial r}\left(r^{2} \frac{\partial}{\partial r}\right)+\frac{\ell(\ell+1)}{2 r^{2}}+v(r)\right) \varphi_{\ell m},
$$

where we have used the fact that

$$
\Lambda \bar{Y}_{\ell m}=-\ell(\ell+1) \bar{Y}_{\ell m} .
$$

Equation (86) shows that the Hamitonian $\hat{H}$ restricted to $\operatorname{Im} P_{m}^{\ell}$ gives rise to the operator

$$
\hat{H}^{\ell}:=-\frac{1}{2} \frac{1}{r^{2}} \frac{\partial}{\partial r}\left(r^{2} \frac{\partial}{\partial r}\right)+\frac{\ell(\ell+1)}{2 r^{2}}+v(r)
$$


which acts on functions of $r$. Here we have denoted the restricted operator by $\hat{H}^{\ell}$ without referring to $m$, since it is independent of $m$, actually. Thus we have obtained reduced quantum systems which are defined on the space given by (81) or (82) together with the reduced Hamiltonian operator $\hat{H}^{\ell}$ given by (88).

In conclusion of this example, we consider what boundary conditions come out on wave functions if those wave functions are assumed to be analytic at the origin. Let $f$ be analytic at the origin. Then $f$ is expressed as

$$
f(\mathbf{x})=\sum_{n=0}^{\infty} \sum_{\substack{i+j+k=n \\
i \geqslant 0, j \geqslant 0, k \geqslant 0}} c_{i j k} x_{1}^{i} x_{2}^{j} x_{3}^{k}=\sum_{n=0}^{\infty} r^{n}\left(Y_{n}^{(n)}+Y_{n-2}^{(n)}+\cdots+\left\{\begin{array}{ll}
Y_{0}^{(n)} & (\text { if } n \text { is even }) \\
Y_{1}^{(n)} & (\text { if } n \text { is odd })
\end{array}\right\}\right),
$$

where $Y_{k}^{(n)}$ are spherical harmonics of degree $k, k=0,2, \ldots, n$ or $k=1,3, \ldots, n$, depending on whether $n$ is even or odd. Here, use has been made of the fact that the space, $P^{n}\left(\mathbf{R}^{3}\right)$, of homogeneous polynomials of degree $n$ is decomposed into the direct sum ${ }^{18}$

$$
P^{n}\left(\mathbf{R}^{3}\right)=H^{n}\left(\mathbf{R}^{3}\right) \oplus r^{2} H^{n-2}\left(\mathbf{R}^{3}\right) \oplus \cdots \oplus\left\{\begin{array}{c}
r^{n} H^{0}\left(\mathbf{R}^{3}\right) \\
r^{n-1} H^{1}\left(\mathbf{R}^{3}\right) \quad(\text { if } n \text { is even }),
\end{array}\right.
$$

where $H^{k}\left(\mathbf{R}^{3}\right)$ denotes the space of solid harmonics of degree $k$, and each spherical harmonic $Y_{k}^{(n)}$ in (89) is expressed as a linear combination of the basis of spherical harmonics, $Y_{k m},|m| \leqslant k$, of degree $k$ :

$$
Y_{k}^{(n)}=\sum_{|m| \leqslant k} c_{m}^{(n)} Y_{k m}
$$

If we rewrite the Taylor series (89) as a Fourier series with respect to spherical harmonics $Y_{\ell m}$, and put together the terms containing spherical harmonics of degree $\ell$, then we obtain

$$
r^{\ell} Y_{\ell}^{(\ell)}+r^{\ell+2} Y_{\ell}^{(\ell+2)}+\cdots=\sum_{|m| \leqslant \ell}\left(r^{\ell} c_{m}^{(\ell)}+r^{\ell+2} c_{m}^{(\ell+2)}+\cdots\right) Y_{\ell m}
$$

This implies that if a quantum state with the angular momentum eigenvalues $\mathbf{J}^{2}=\ell(\ell+1)$ and $J_{3}=m$ is analytic at the origin, it is $Y_{\ell m}$ times an analytic function of $r$ which has the term of the lowest order $\ell$ and those of every other higher order. This fact was pointed out in Ref. 1 with the assumption that $v(r)$ is analytic at $r=0$. Our conclusion holds true if $v(r)$ is not analytic at $r$ $=0$, as long as a wave function analytic at the origin is admitted as a quantum state.

We turn to an example of the reduction by a finite group. Since we shall deal with a nontrivial application of it in the next section, we give here a quite simple example. Let $M=\mathbf{R}^{n}$ and $H$ $=\mathbf{Z}_{2}=\{ \pm 1\}$. The group $\mathbf{Z}_{2}$ acts on $\mathbf{R}^{n}$ in the manner

$$
x \mapsto \varepsilon x, \quad x \in \mathbf{R}^{n}, \quad \varepsilon \in \mathbf{Z}_{2} .
$$

All the inequivalent unitary irreducible representations are the trivial representation, $\varepsilon \mapsto 1$, and the tautological representation, $\varepsilon \mapsto \varepsilon$. Then the Fourier series expansion (36) becomes simply

$$
f(x)=\frac{1}{2}(f(x)+f(-x))+\frac{1}{2}(f(x)-f(-x)) .
$$

\section{APPLICATION TO MANY-PARTICLE SYSTEMS}

We consider a system of many particles lying in $\mathbf{R}^{3}$. Let $\mathbf{x}_{1}, \ldots, \mathbf{x}_{N}$ be position vectors of particles and $m_{1}, \ldots, m_{N}$ their masses. Let $M$ be the center-of-mass system, which is defined to be 


$$
M=\left\{\begin{array}{l|l}
x=\left(\mathbf{x}_{1}, \ldots, \mathbf{x}_{N}\right) & \sum_{i=1}^{N} m_{i} \mathbf{x}_{i}=0
\end{array},\right.
$$

and isomorphic to $\mathbf{R}^{3(N-1)}$ as a vector space. The rotation group $\mathrm{SO}(3)$ acts on $M$ in such a manner that

$$
\left(\mathbf{x}_{1}, \ldots, \mathbf{x}_{N}\right) \mapsto\left(g \mathbf{x}_{1}, \ldots, g \mathbf{x}_{N}\right), \quad g \in \mathrm{SO}(3)
$$

The configurations of particles are characterized by the linear subspaces

$$
F_{x}:=\operatorname{span}\left\{\mathbf{x}_{1}, \mathbf{x}_{2}, \ldots, \mathbf{x}_{N}\right\}, \quad x \in M
$$

According to $\operatorname{dim} F_{x}=0,1,2,3$, the configurations $x$ are pointlike, collinear, planar, and spatial, respectively. Let $M_{k}, k=0,1,2,3$, denote the space of respective configurations of particles. Then $M$ is broken up into

$$
M=\bigcup_{k=0}^{3} M_{k}, \quad M_{k}:=\left\{x \in M \mid \operatorname{dim} F_{x}=k\right\} .
$$

One can show that $\mathrm{SO}(3)$ acts on $\dot{M}:=M_{2} \cup M_{3}$ freely, that is, if $g x=x$ for some $x$ $\in M_{2} \cup M_{3}$, then $g=I$ (the $3 \times 3$ identity matrix). This means that the isotropy subgroup is trivial at every point of $\dot{M}$, that is, $G_{x}=\{e\}$ for $x \in \dot{M}$. However, the isotropy subgroups $G_{x}$ at $x \in M_{1}$ and at $x \in M_{0}$ are isomorphic with $\mathrm{SO}(2)$ and with $\mathrm{SO}(3)$, respectively. On restricting $M$ to $\dot{M}$, we can make $\dot{M}$ into a principal fiber bundle $\dot{M} \rightarrow \dot{M} / \mathrm{SO}(3)$. However, the total space $M$ cannot be made into a fiber bundle. This is because one has "singular" orbits of SO(3) through points outside of $\dot{M}$; the orbits through each point of $M_{1}$ and of $M_{0}$ are diffeomorphic with $S^{2}$ $=\mathrm{SO}(3) / \mathrm{SO}(2)$ and with a point, respectively, while generic orbits through $x \in \dot{M}$ are diffeomorphic with $\mathrm{SO}(3)$.

Since a Hamiltonian $\hat{H}$ for many-particle systems with internal interaction only is $\mathrm{SO}(3)$ invariant, the reduction procedure with compact Lie groups is applied to provide a reduced system $\left(L^{2}\left(M ; \mathcal{H}^{\ell}\right)^{G}, \operatorname{id}_{\mathcal{H}} \ell \otimes \hat{H}\right)$ with $G=\mathrm{SO}(3)$ and $\ell=0,1,2, \ldots$. Note that at this level of reduction, $M$ does not need to be restricted to $\dot{M}$. Equation (19) then takes the form

$$
\left(E_{m}^{\ell} f\right)(g x)=D^{\ell}(g)\left(E_{m}^{\ell} f\right)(x), \quad g \in \mathrm{SO}(3), x \in M,
$$

which implies that the $\mathcal{H}^{\ell}$-valued function $E_{m}^{\ell} f$ describes an eigenstate associated with the eigenvalue $\ell(\ell+1)$ of the total angular momentum operator.

Restricting $M$ to $\dot{M}$, we obtain a principal bundle $\dot{M} \rightarrow \dot{M} / \mathrm{SO}(3)$ and can make up the vector bundle associated with $\dot{M} \rightarrow \dot{M} / \mathrm{SO}(3)$ by using a representation space $\mathcal{H}^{\ell}$. The reduced system $\left(L^{2}\left(\dot{M} ; \mathcal{H}^{\ell}\right)^{\mathrm{SO}(3)}, \mathrm{id}_{\mathcal{H}_{\ell}} \otimes \hat{H}\right)$ is then in one-to-one correspondence with $\left(\Gamma_{\ell}^{2}(\dot{M} / \mathrm{SO}(3)), \hat{H}^{\ell}\right)$. If we want to treat the whole of $M$, we must impose boundary conditions on wave functions at the boundary $\partial \dot{M}=M_{0} \cup M_{1} \cdot{ }^{4}$ Since the collinear configurations are in $M_{1}$, we have to consider boundary conditions on wave functions at collinear configurations. For a three-body system, Mitchell and Littlejohn ${ }^{19}$ studied the behavior of wave functions at collinear configurations from the viewpoint of bundle theory. By a coordinate-based method, Watson ${ }^{20}$ studied small vibrations in the neighborhood of collinear configurations.

We turn to the reduction of quantum systems with discrete symmetry. The center-of-mass system $M$ is looked upon as the set of configurations of the Jacobi vectors $\left(\mathbf{r}_{1}, \ldots, \mathbf{r}_{N-1}\right)$, where $\mathbf{r}_{j}$ 's are defined to be 


$$
\mathbf{r}_{j}=\left(\frac{1}{\mu_{j}}+\frac{1}{m_{j+1}}\right)^{-1 / 2}\left(\mathbf{x}_{j+1}-\frac{1}{\mu_{j}} \sum_{i=1}^{j} m_{i} \mathbf{x}_{i}\right), \quad \mu_{j}=\sum_{i=1}^{j} m_{i} .
$$

We here assume that all particles are identical and set the masses all equal to one. Then Eq. (100) becomes

$$
\mathbf{r}_{j}=\sqrt{\frac{j}{j+1}}\left(\mathbf{x}_{j+1}-\frac{1}{j} \sum_{i=1}^{j} \mathbf{x}_{i}\right)
$$

Since all particles are identical, the system is unchanged if particles are exchanged mutually. Put another way, the configurations of particles admits symmetry by the action of the symmetric group $S_{N}$

$$
\left(\mathbf{x}_{1}, \ldots, \mathbf{x}_{N}\right) \mapsto\left(\mathbf{x}_{\sigma(1)}, \ldots, \mathbf{x}_{\sigma(N)}\right), \quad \sigma \in S_{N}
$$

Since new Jacobi vectors associated with a new configuration $\left(\mathbf{x}_{\sigma(1)}, \ldots, \mathbf{x}_{\sigma(N)}\right)$,

$$
\mathbf{r}_{j}^{\sigma}=\sqrt{\frac{j}{j+1}}\left(\mathbf{x}_{\sigma(j+1)}-\frac{1}{j} \sum_{i=1}^{j} \mathbf{x}_{\sigma(i)}\right), \quad j=1, \ldots, N-1,
$$

are linearly related with the old Jacobi vectors $\mathbf{r}_{j}$, there exists an $N \times N$ matrix $A$ depending on $\sigma \in S_{N}$ such that $\left(\mathbf{r}_{1}^{\sigma}, \ldots, \mathbf{r}_{N-1}^{\sigma}\right)=\left(\mathbf{r}_{1}, \ldots, \mathbf{r}_{N-1}\right) A$. Thus one can find a matrix representation $\pi$ : $S_{N} \rightarrow G L(N-1, \mathbf{R})$ through $\pi\left(\sigma^{-1}\right)=A$. Thus, $S_{N}$ acts on $M$ in the manner

$$
\left(\mathbf{r}_{1}, \ldots, \mathbf{r}_{N-1}\right) \mapsto\left(\mathbf{r}_{1}, \ldots, \mathbf{r}_{N-1}\right) \pi\left(\sigma^{-1}\right), \quad \sigma \in S_{N} .
$$

We have to note here that since we deal with $S_{N}$ as acting on $M$ to the right, the product $\sigma \tau$ of $\sigma$, $\tau \in S_{N}$ is interpreted as this: $\sigma$ comes first and then $\tau$ follows, so that one has $\left(\begin{array}{llll}1 & 2\end{array}\right)\left(\begin{array}{lll}1 & 2 & 3\end{array}\right)$ $=\left(\begin{array}{ll}1 & 3\end{array}\right)$, for example. If we choose the left action of $S_{N}$, we shall obtain $\left(\begin{array}{ll}1 & 2\end{array}\right)\left(\begin{array}{ll}1 & 2\end{array} 3\right)=\left(\begin{array}{ll}2 & 3\end{array}\right)$, of course.

For example, if $N=3$, one obtains the two-dimensional representation $\pi^{2}$ which has the matrix expression as follows:

$$
\begin{gathered}
\pi^{2}\left(\sigma_{1}\right)=\left(\begin{array}{ll}
1 & 0 \\
0 & 1
\end{array}\right), \quad \pi^{2}\left(\sigma_{4}\right)=\left(\begin{array}{cc}
-1 & 0 \\
0 & 1
\end{array}\right), \\
\pi^{2}\left(\sigma_{2}\right)=\left(\begin{array}{cc}
-1 / 2 & \sqrt{3} / 2 \\
-\sqrt{3} / 2 & -1 / 2
\end{array}\right), \quad \pi^{2}\left(\sigma_{5}\right)=\left(\begin{array}{cc}
1 / 2 & \sqrt{3} / 2 \\
\sqrt{3} / 2 & -1 / 2
\end{array}\right), \\
\pi^{2}\left(\sigma_{3}\right)=\left(\begin{array}{cc}
-1 / 2 & -\sqrt{3} / 2 \\
\sqrt{3} / 2 & -1 / 2
\end{array}\right), \quad \pi^{2}\left(\sigma_{6}\right)=\left(\begin{array}{cc}
1 / 2 & -\sqrt{3} / 2 \\
-\sqrt{3} / 2 & -1 / 2
\end{array}\right),
\end{gathered}
$$

where

$$
\begin{gathered}
\sigma_{1}=(1), \quad \sigma_{2}=\left(\begin{array}{lll}
1 & 2 & 3
\end{array}\right), \quad \sigma_{3}=\left(\begin{array}{lll}
1 & 3 & 2
\end{array}\right), \\
\sigma_{4}=\left(\begin{array}{ll}
1 & 2
\end{array}\right), \quad \sigma_{5}=\left(\begin{array}{ll}
2 & 3
\end{array}\right), \quad \sigma_{6}=\left(\begin{array}{ll}
1 & 3
\end{array}\right) .
\end{gathered}
$$

Incidentally, it is well known that there are three inequivalent unitary irreducible representations of $S_{3}$. The $\pi^{2}$ is one of them, and the other two are the trivial representation: $\pi^{0}(\sigma)=1$, and the signum representation; $\pi^{1}(\sigma)=\operatorname{sgn}(\sigma)$, both of which are one-dimensional representations. According to the Fourier series expansion formula (36), $f \in L^{2}(M)$ is decomposed into 


$$
f(x)=\left(Q_{1}^{0} f\right)(x)+\left(Q_{1}^{1} f\right)(x)+\sum_{j=1}^{2}\left(Q_{j}^{2} f\right)(x),
$$

where $Q_{j}^{\ell}:=Q_{j}^{\pi^{\ell}}, \ell=0,1,2, j=1, \ldots, d^{\pi^{\ell}}$, are the projection operators defined by (34). Denoting $\left(\mathbf{r}_{1}, \mathbf{r}_{2}\right) \pi^{2}\left(\sigma_{\alpha}^{-1}\right), \alpha=1, \ldots, 6$, simply by $x \sigma_{\alpha}$, we can show that

$$
\begin{gathered}
\left(Q_{1}^{0} f\right)(x)=\frac{1}{6}\left(f\left(x \sigma_{1}\right)+f\left(x \sigma_{2}\right)+f\left(x \sigma_{3}\right)+f\left(x \sigma_{4}\right)+f\left(x \sigma_{5}\right)+f\left(x \sigma_{6}\right)\right), \\
\left(Q_{1}^{1} f\right)(x)=\frac{1}{6}\left(f\left(x \sigma_{1}\right)+f\left(x \sigma_{2}\right)+f\left(x \sigma_{3}\right)-f\left(x \sigma_{4}\right)-f\left(x \sigma_{5}\right)-f\left(x \sigma_{6}\right)\right), \\
\left(Q_{1}^{2} f\right)(x)=\frac{1}{3}\left(f\left(x \sigma_{1}\right)-\frac{1}{2} f\left(x \sigma_{2}\right)-\frac{1}{2} f\left(x \sigma_{3}\right)-f\left(x \sigma_{4}\right)+\frac{1}{2} f\left(x \sigma_{5}\right)+\frac{1}{2} f\left(x \sigma_{6}\right)\right), \\
\left.\left(Q_{2}^{2} f\right)(x)=\frac{1}{3} f\left(x \sigma_{1}\right)-\frac{1}{2} f\left(x \sigma_{2}\right)-\frac{1}{2} f\left(x \sigma_{3}\right)+f\left(x \sigma_{4}\right)-\frac{1}{2} f\left(x \sigma_{5}\right)-\frac{1}{2} f\left(x \sigma_{6}\right)\right) .
\end{gathered}
$$

We proceed to the reduction of quantum systems of many identical particles by $S_{N}$. Since the Hamiltonian $\hat{H}$ should be permutation invariant, we can apply the reduction procedure with finite groups to obtain $\left(L^{2}\left(M ; \mathcal{K}^{\chi}\right)^{H}, \operatorname{id}_{\mathcal{K} \chi} \otimes \hat{H}\right)$ with $H=S_{N}$. As for $S_{N}$, we have two representations frequently used in many-identical particle systems, one of which is a trivial representation, $\pi^{0}$ : $g \mapsto 1$, and the other the signum representation, $\pi^{1}: g \mapsto \operatorname{sgn}(g)$, where $\operatorname{sgn}(\sigma)$ is equal to 1 or -1 according to whether $\sigma$ is an even or odd permutation. For $\pi^{0}$ and $\pi^{1}$, the projection operators defined in (34) take the form

$$
Q^{0}=\frac{1}{N !} \sum_{\sigma \in S_{N}} V(\sigma) \quad \text { and } \quad Q^{1}=\frac{1}{N !} \sum_{\sigma \in S_{N}} \operatorname{sgn}(\sigma) V(\sigma)
$$

respectively, where we have denoted $Q_{1}^{0}$ and $Q_{1}^{1}$ simply by $Q^{0}$ and $Q^{1}$, respectively, as $\pi^{0}$ and $\pi^{1}$ are one-dimensional representations. The operators $Q^{0}$ and $Q^{1}$ provide a method for forming wave functions obeying Bose and Fermi statistics, respectively. In fact, from (46) with $H=S_{N}$, one obtains

$$
\left(Q^{0} f\right)(x g)=\left(Q^{0} f\right)(x), \quad\left(Q^{1} f\right)(x g)=\operatorname{sgn}(g)\left(Q^{1} f\right)(x), \quad g \in S_{N} .
$$

Note here that one has $F_{1}^{0}=Q^{0}$ and $F_{1}^{1}=Q^{0}$, since $\pi^{0}$ and $\pi^{1}$ are one-dimensional representations. Thus Bose and Fermi statistics are viewed as $\pi^{0}$-equivariant and $\pi^{1}$-equivariant states, respectively, so that they are considered as reduced states with respect to $S_{N}$.

To give another reduced state, we consider the system of three identical particles and the representation $\pi^{2}$ given by (105). Then we can form $\mathbf{C}^{2}$-valued $\pi^{2}$-equivariant functions $F_{j}^{2} f$ which obey the transformation rule coming from (46):

$$
\left(F_{j}^{2} f\right)(x g)=\pi^{2}(g)^{T}\left(F_{j}^{2} f\right)(x), \quad j=1,2, \quad x \in M .
$$

Since (113) is a generalization of (112) with $N=3$, we may consider that $F_{j}^{2} f$ obeys some kind of statistics, like Bose or Fermi statistics. The $\mathbf{C}^{2}$-valued equivariant functions are described explicitly as follows:

$$
\left(F_{1}^{2} f\right)(x)=\frac{1}{\sqrt{2}}\left(\begin{array}{c}
\left(Q_{11}^{2} f\right)(x) \\
\left(Q_{21}^{2} f\right)(x)
\end{array}\right), \quad\left(F_{2}^{2} f\right)(x)=\frac{1}{\sqrt{2}}\left(\begin{array}{c}
\left(Q_{12}^{2} f\right)(x) \\
\left(Q_{22}^{2} f\right)(x)
\end{array}\right)
$$

where 


$$
\begin{aligned}
& \left(Q_{21}^{2} f\right)(x)=\frac{1}{3}\left(-\frac{\sqrt{3}}{2} f\left(x \sigma_{2}\right)+\frac{\sqrt{3}}{2} f\left(x \sigma_{3}\right)+\frac{\sqrt{3}}{2} f\left(x \sigma_{5}\right)-\frac{\sqrt{3}}{2} f\left(x \sigma_{6}\right)\right), \\
& \left(Q_{12}^{2} f\right)(x)=\frac{1}{3}\left(\frac{\sqrt{3}}{2} f\left(x \sigma_{2}\right)-\frac{\sqrt{3}}{2} f\left(x \sigma_{3}\right)+\frac{\sqrt{3}}{2} f\left(x \sigma_{5}\right)-\frac{\sqrt{3}}{2} f\left(x \sigma_{6}\right)\right)
\end{aligned}
$$

and $Q_{11}^{2}=Q_{1}^{2}$ and $Q_{22}^{2}=Q_{1}^{2}$ are given in (110). We notice in addition that terms containing $f\left(x \sigma_{1}\right)$ and $f\left(x \sigma_{4}\right)$ disappear on the right-hand sides of (115) on account of vanishing coefficients $\pi_{21}^{2}\left(\sigma_{1}\right)=\pi_{21}^{2}\left(\sigma_{4}\right)=0$, etc.

In conclusion, we have to point out that the action (104) of $S_{N}$ on the center-of-mass system $M$ determines an $(N-1)$-dimensional unitary representation of $S_{N}$. For example, for $N=4$, we can show, by the help of computer algebra, that the representation determined by (104) has the matrix expression as follows:

$$
\begin{aligned}
& \pi(1)^{-1}=\left(\begin{array}{lll}
1 & 0 & 0 \\
0 & 1 & 0 \\
0 & 0 & 1
\end{array}\right), \\
& \pi(123)^{-1}=\left(\begin{array}{ccc}
-\frac{1}{2} & -\frac{1}{2} \sqrt{3} & 0 \\
\frac{1}{2} \sqrt{3} & -\frac{1}{2} & 0 \\
0 & 0 & 1
\end{array}\right) \text {, } \\
& \pi(124)^{-1}=\left(\begin{array}{ccc}
-\frac{1}{2} & -\frac{1}{6} \sqrt{3} & -\frac{1}{3} \sqrt{6} \\
\frac{1}{6} \sqrt{3} & \frac{5}{6} & -\frac{1}{3} \sqrt{2} \\
\frac{1}{3} \sqrt{6} & -\frac{1}{3} \sqrt{2} & -\frac{1}{3}
\end{array}\right), \\
& \pi(132)^{-1}=\left(\begin{array}{ccc}
-\frac{1}{2} & \frac{1}{2} \sqrt{3} & 0 \\
-\frac{1}{2} \sqrt{3} & -\frac{1}{2} & 0 \\
0 & 0 & 1
\end{array}\right) \text {, } \\
& \pi(134)^{-1}=\left(\begin{array}{ccc}
\frac{1}{2} & -\frac{1}{6} \sqrt{3} & -\frac{1}{3} \sqrt{6} \\
-\frac{1}{2} \sqrt{3} & -\frac{1}{6} & -\frac{1}{3} \sqrt{2} \\
0 & \frac{2}{3} \sqrt{2} & -\frac{1}{3}
\end{array}\right) \text {, }
\end{aligned}
$$




$$
\pi(142)^{-1}=\left(\begin{array}{ccc}
-\frac{1}{2} & \frac{1}{6} \sqrt{3} & \frac{1}{3} \sqrt{6} \\
-\frac{1}{6} \sqrt{3} & \frac{5}{6} & -\frac{1}{3} \sqrt{2} \\
-\frac{1}{3} \sqrt{6} & -\frac{1}{3} \sqrt{2} & -\frac{1}{3}
\end{array}\right),
$$




$$
\pi((14)(23))^{-1}=\left(\begin{array}{ccc}
0 & \frac{1}{3} \sqrt{3} & -\frac{1}{3} \sqrt{6} \\
\frac{1}{3} \sqrt{3} & -\frac{2}{3} & -\frac{1}{3} \sqrt{2} \\
-\frac{1}{3} \sqrt{6} & -\frac{1}{3} \sqrt{2} & -\frac{1}{3}
\end{array}\right),
$$




$$
\pi(1234)^{-1}=\left(\begin{array}{ccc}
-\frac{1}{2} & -\frac{1}{6} \sqrt{3} & -\frac{1}{3} \sqrt{6} \\
\frac{1}{2} \sqrt{3} & -\frac{1}{6} & -\frac{1}{3} \sqrt{2} \\
0 & \frac{2}{3} \sqrt{2} & -\frac{1}{3}
\end{array}\right),
$$

It is known that there are two three-dimensional inequivalent unitary representations of $S_{4}$, one of which is isomorphic with the group of symmetries of the tetrahedron, and the other with the group 
of symmetries of the cube. ${ }^{18}$ The former is a discrete subgroup of $\mathrm{O}(3)$ and the latter a discrete subgroup of $\mathrm{SO}(3)$. Since the group $\pi\left(S_{4}\right)$ given above includes matrices of determinant -1 , it must be isomorphic with the group of symmetries of the tetrahedron.

In conclusion, we note that since the actions of $\mathrm{SO}(3)$ and $S_{N}$ commute, one can perform the reduction procedure with $\mathrm{SO}(3)$ and further with $S_{N}$, so that one can talk about Bose and Fermi statistics for the reduced states in $\left(\Gamma_{\ell}^{2}\left(\dot{M} / \mathrm{SO}(3) ; \mathcal{H}^{\ell}\right)\right.$.

\section{ACKNOWLEDGMENTS}

This work was partly supported by the Grant-in-Aid for the Scientific Research of the Ministry of Education, Culture, Sports, Science and Technology of Japan (No. 13640209).

\footnotetext{
${ }^{1}$ A. Messiah, Quantum Mechanics (North-Holland, Amsterdam, 1965), Vols. I and II.

${ }^{2}$ T. Bröcker and T. tom Dieck, Representations of Compact Lie Groups (Springer-Verlag, New York, 1985), for example. ${ }^{3}$ A. Terras, Fourier Analysis on Finite Groups and Applications (Cambridge University Press, Cambridge, 1999), for example.

${ }^{4}$ S. Tanimura and T. Iwai, J. Math. Phys. 41, 1814 (2000).

${ }^{5}$ A. Guichardet, Ann. I.H.P. Phys. Theor. 40, 329 (1984).

${ }^{6}$ A. Tachibana and T. Iwai, Phys. Rev. A 33, 2262 (1986).

${ }^{7}$ R. Montgomery, in The Geometry of Hamiltonian Systems, edited by T. Ratiu (Springer-Verlag, New York, 1991), p. 403.

${ }^{8}$ R. Montgomery, in Dynamics and Control of Mechanical Systems, edited by M. J. Enos (American Mathematics Society, Providence, RI, 1993), p. 193.

${ }^{9}$ T. Iwai, J. Math. Phys. 28, 964 (1987).

${ }^{10}$ T. Iwai, J. Math. Phys. 28, 1315 (1987).

${ }^{11}$ T. Iwai, Ann. I.H.P. Phys. Theor. 47, 199 (1987).

${ }^{12}$ T. Iwai, J. Math. Phys. 29, 1325 (1988).

${ }^{13}$ T. Iwai, J. Phys. A 31, 3849 (1998).

${ }^{14}$ T. Iwai, J. Math. Phys. 40, 2381 (1999).

${ }^{15}$ R. G. Littlejohn and M. Reinsch, Rev. Mod. Phys. 69, 213 (1997).

${ }^{16}$ G. S. Ezra, Symmetry Properties of Molecules, Lecture Notes in Chemistry Vol. 28 (Springer, New York, 1982).

${ }^{17}$ L. C. Biedenharn and J. D. Louck, Angular Momentum in Quantum Physics, Encyclopedia of Mathematics and Its Applications, Vol. 8 (Addison-Wesley, Reading, MA, 1981), for example.

${ }^{18}$ S. Sternberg, Group Theory and Physics (Cambridge University Press, Cambridge, 1994), for example.

${ }^{19}$ K. A. Mitchell and R. G. Littlejohn, Phys. Rev. A 61, 042502 (2000).

${ }^{20}$ K. Watson, Mol. Phys. 19, 465 (1970).
} 